\title{
Band Structure Engineering in Sn1.03Te Through In-induced Resonant Level
}

Shantanu Misra1, Bartlomiej Wiendlocha2, $\uparrow$, Janusz Tobola2, Florian Fesquet1, Anne

Dauscher1, Bertrand Lenoir1, Christophe Candolfi1,*

IInstitut Jean Lamour, UMR 7198 CNRS - Université de Lorraine, 2 allée André Guinier-

Campus ARTEM, BP 50840, 54011 Nancy Cedex, France

2 Faculty of Physics and Applied Computer Science, AGH University of Science and

Technology, Aleja Mickiewicza 30, 30-059 Krakow, Poland

*Corresponding authors:*christophe.candolfi@univ-lorraine.fr ; † wiendlocha@ fis.agh.edu.pl

\begin{abstract}
Narrow-band-gap IV-VI semiconductors represent a historically important class of thermoelectric materials. As one of the representative compound of this class, SnTe has been reinvestigated over the last years demonstrating its potential as a high-temperature $p$-type thermoelectric material. Here, we present a detailed study of the influence of very low doping levels of In, from $0.05 \%$ up to $2 \%$, on the high-temperature transport properties of the selfcompensated Sn1.03Te compound. Our results evidence a strong impact of In on the transport properties, consistent with the presence of an In-induced resonant level (RL) in the valence bands of Sn1.03Te. This peculiar behavior is confirmed by electronic band structure calculations performed using the Korringa-Kohn-Rostoker method with the coherent potential approximation (KKR-CPA) revealing a narrow and sharp peak in the density of states (DOS) induced by the hybridization of the In $s$-states with the electronic states of $\mathrm{Sn}_{1.03} \mathrm{Te}$. This
\end{abstract}


distortion in the DOS results in a spectacular increase in both the thermopower and electrical resistivity at $300 \mathrm{~K}$. Although the influence of the RL is somewhat lessened at higher temperatures, a significant enhancement in the $Z T$ values is nevertheless achieved with a peak $Z T$ of 0.75 at $800 \mathrm{~K}$ which represents an increase of $35 \%$ over the values measured in $\mathrm{Sn} 1.03 \mathrm{Te}$. Of relevance for practical applications, the weak dependence of the RL on temperature leads to enhanced average $Z T$ value.

\section{Introduction}

Thermoelectric materials constitute a class of compounds that can convert a thermal gradient into an electric potential and vice versa, allowing for any waste heat to be harvested into useful electrical energy.1,2 The efficiency with which this energy conversion is realized is mainly determined by the material's dimensionless thermoelectric figure of merit defined as $Z T=\alpha^{2} T / \rho\left(\kappa_{e}+\kappa_{p h}\right)=P T /\left(\kappa_{e}+\kappa_{p h}\right)$ where $\alpha$ is the thermopower (or Seebeck coefficient), $T$ is the absolute temperature, $\rho$ is the electrical resistivity, $P=\alpha^{2} / \rho$ is the power factor and $\kappa_{e}$ and $\kappa_{p h}$ are the electronic and lattice thermal conductivity, respectively.1,2 Increasing the efficiency of a thermoelectric device relies on designing materials with high $Z T$ values at the operating temperature. The optimization of this material's parameter is however notoriously challenging due to the intertwined nature of the above-mentioned transport properties. Most strategies developed can be divided into two main categories: the optimization of the power factor through band structure engineering and the minimization of the phononmediated heat transport.2

One possible way of optimizing the power factor is via the introduction of an impurity that gives rise to a so-called resonant level (RL) at the edge of either the valence or conduction bands.3-5 The impurity atoms distort locally the electronic band structure beyond a rigid-band 
model. A significant increase in the thermopower and hence, in the power factor is then observed when the chemical potential resides in this distortion due to the increased density of states (DOS). In addition, a subtle balance between the localized nature of the RL and its hybridization with the states of the host structure must be achieved. This requires a proper selection of the resonant impurity, as well as its concentration. For example, a $3 d$ transition metal impurity in IV-VI semiconductors may form a large DOS peak. However, due to a poor hybridization with the $s-p$ host electronic states, the resonant level tends to localize by forming a narrow impurity band. On the other hand, too large concentration of the $s$-p-like RL may lead to too large smearing of the resonant level, suppressing its beneficial influence on the thermopower. Examples of these two extreme cases are provided by the PbTe:Ti and Sn-doped $\mathrm{Bi}_{2} \mathrm{Te} 3$ systems, respectively.6-9

The presence of a RL has been demonstrated in several chalcogenide compounds, most of them classified as state-of-the-art thermoelectric materials. Prominent examples include Sndoped $\mathrm{Bi} 2 \mathrm{Te}$, Tl-doped PbTe or, more recently, Sn-doped $\alpha$-As2Te3.3,5,8-10 P-type SnTe has been intensively reinvestigated over the last years as a $\mathrm{Pb}$-free analogue of $\mathrm{PbTe}$ with hightemperature thermoelectric properties rivaling those achieved in $\mathrm{PbTe}$ upon proper optimization through substitutions, nanostructuration or a combination of both.11-32 This compound recently entered the above-mentioned list of known RL with the identification of a RL induced by In substitution on the Sn site.33 Intriguingly, In induces superconductivity over a wide range of concentrations with critical temperatures as high as $4.5 \mathrm{~K}, 34-45$ bearing resemblance with the anomalous, low-carrier-density superconductor $\mathrm{Pb}_{1-x} \mathrm{Tl} \mathrm{l}_{x} \mathrm{Te} .46-48$ The possible connection of the In resonant behavior in SnTe with superconductivity is however yet to be determined.

The initial investigation of the high-temperature thermoelectric properties of the series $\operatorname{Sn} 1-x \operatorname{In} x$ Te for $x=0.0025,0.005$ and 0.01 identified the lowest In content studied as the optimum concentration.33 Combined with reduced lattice thermal conductivity achieved by ball-milling, 
the resonant-driven enhancement of the power factor led to an improved $Z T$ value of 1.1 at 873 K.33 Compared to other RL systems for which the optimum doping level is typically around $2 \%$, this In concentration is significantly lower, possibly due to the large amount of Sn vacancies inherently present in pristine SnTe. Because the heavy hole doping induced by these vacancies may shift the chemical potential away from the RL, it is of interest to determine in more detail their influence on the thermoelectric properties of In-substituted SnTe. The introduction of excess $\mathrm{Sn}$ in starting compositions has been widely shown to be an effective way of reducing the vacancy concentration in SnTe, making the series $\mathrm{Sn}_{1.03-x \operatorname{In} x} \mathrm{Te}$ worthy of further experimental and theoretical investigation.

Here, we investigate in detail the combined impact of Sn self-compensation and In substitution on the high-temperature thermoelectric properties of $\operatorname{Sn}_{1.03-x} \operatorname{In} x$ Te by electronic band structure calculations coupled with transport properties measurements $(300-800 \mathrm{~K})$. Through systematic variations in the In content from $x=0.0005$ up to 0.02 , we observe a stepwise increase in the $\alpha$ and $\rho$ values at $300 \mathrm{~K}$ which evidences the strong impact of In on the transport properties despite the very low concentrations probed. A careful comparison of the experimental data with the Ioffe-Pisarenko plot, predicting the hole dependence of $\alpha$, confirms that In also acts as a resonant impurity in $\mathrm{Sn}_{1.03} \mathrm{Te}$. The excess $\mathrm{Sn}$ introduced leads to a shift of the optimum In concentration at which the highest thermoelectric properties are achieved. Although the beneficial influence of the resonance is lessened at high temperatures, the Ininduced RL yields a peak $Z T$ value of 0.75 at $800 \mathrm{~K}$ for $x=0.0035$, which represents an improvement of $35 \%$ compared to the self-compensated sample Sn1.03Te. 


\section{Experimental and computational details}

\subsection{Synthesis and sample characterization}

Polycrystalline samples $\operatorname{Sn} 1.03-x \operatorname{In} x \operatorname{Te}(x=0,0.0005,0.0015,0.0025,0.0035,0.0045,0.0075$, 0.01 and 0.02 ) were prepared by powder metallurgy using stoichiometric quantities of Sn shots (99.999\%), In shots (99.999\%) and Te powders (99.999\%). Prior to use, both Sn and Te were purified to eliminate possible traces of oxygen. These starting elements were placed in evacuated silica tubes sealed under secondary vacuum. Due to the high vapor pressure of SnTe at high temperatures that may influence the actual chemical compositions, all samples were carefully prepared in silica ampules of similar dimensions to ensure a nearly equivalent sampleto-volume ratio. The sealed ampules were heated in a rocking furnace to $1100 \mathrm{~K}$, kept at this temperature for $5 \mathrm{~h}$ and then quenched in room-temperature water. The ingots were handground into fine powders that were consolidated by spark plasma sintering in graphite dies of $10 \mathrm{~mm}$ in diameter at $750 \mathrm{~K}$ under a uniaxial pressure of $65 \mathrm{MPa}$ for 10 minutes. All the resulting cylindrical pellets were found to have geometrical densities exceeding $96 \%$ of the theoretical density.

Powder X-ray diffraction (PXRD) was performed at room temperature to check the crystal structure and the possible presence of secondary phases using a Bruker D8 Advance diffractometer ( $\mathrm{CuK} \alpha 1$ radiation).

\subsection{Transport property measurements}

The consolidated pellets were cut with a diamond-wire saw into bar-shaped $(\sim 1.5 \times 1.5 \times 8 \mathrm{~mm} 3)$ and disc-shaped $(\sim 10 \mathrm{~mm}$ in diameter, $\sim 1 \mathrm{~mm}$ in thickness $)$ samples. 
Hall effect measurements were performed at $300 \mathrm{~K}$ with a five-probe configuration using the ac transport option of a physical property measurement system (PPMS, Quantum Design). Good electrical contacts were realized on the bar-shaped samples by brazing copper wires onto the samples with a low-melting point braze. The Hall resistivity $\rho_{H}$ was determined by measuring the antisymmetric component of the transverse $\rho_{x y}$ voltage under magnetic field reversal following the formula $\rho_{H}=\left[\rho_{x y}\left(+\mu_{0} H\right)-\rho_{x y}\left(-\mu_{0} H\right)\right] / 2$. The Hall coefficient $R_{H}$ was derived from the slope of the $\rho_{H}\left(\mu_{0} H\right)$ data for fields $-1 \leq \mu_{0} H \leq 1 \mathrm{~T}$. The Hall hole concentration $p$ and the Hall mobility $\mu_{H}$ were determined from the single-carrier relations $p=$ $1 / R_{H} e$ and $\mu_{H}=R_{H} / \rho$ where $e$ is the elemental charge.

The electrical resistivity and thermopower were simultaneously measured on bar-shaped samples between 300 and $800 \mathrm{~K}$ by a conventional four-probe method under a low helium pressure. The thermal diffusivity $a$ was measured on the disc-shaped sample between 300 and $800 \mathrm{~K}$ with a Netzsch laser flash instrument (LFA 467) under continuous argon flow. Prior to measurement, the samples were spray-coated with a thin layer of graphite to ensure a homogeneous signal absorption and emission on the respective surfaces. The thermal diffusivity was used to calculate the total thermal conductivity $\kappa$ via the formula $\kappa=a C_{p} d$ where $C_{p}$ is the specific heat and $d$ is the experimental density determined from the weight and geometric dimensions of the consolidated pellets. $C_{p}$ was approximated by the Dulong-Petit law $C_{p}=3 N R$ where $N$ is the number of atoms per formula unit and $R$ is the gas constant. The temperature dependence of the density was not taken into account in the present case.

\subsection{Electronic band structure calculations}

Electronic structure calculations were performed using the Korringa-Kohn-Rostoker (KKR) method with the coherent potential approximation (CPA), applied to account for the chemical 
disorder,49,50 as implemented in the Munich SPR-KKR package.51 The experimental rock-salt crystal structure and lattice parameter $(6.32 \AA)$ were used. Regular $k$-point mesh was used in calculations, using $1500-5000 k$-points for the self-consistent cycle and $4-20 \times 105$ for the density of states (DOS) and Bloch spectral density functions (BSF) calculations (number of points given in the irreducible part of the Brillouin zone). The crystal potential was constructed in the framework of the local density approximation (LDA), using Vosko, Wilk and Nussair formula for the exchange-correlation part.52 For all atoms, angular momentum cut-off $l_{\max }=3$ was set and full-potential full-relativistic calculations were performed. High convergence limits were put on the self-consistent cycle (10-5 Ry for the Fermi level $E_{F}$ and for the total energy). The position of $E_{F}$ was obtained using the Lloyd formula.49 To analyze the influence of the impurities on the electronic bands, Bloch spectral density functions were computed. This technique allows to take into account the effect of electron scattering, due to presence of impurities, on the electronic structure, which can be intuitively visualized by plotting the smeared electronic bands. This approach has been already successfully applied to study the influence of resonant impurities on the band structure of several thermoelectric materials, such as $\mathrm{PbTe}: \mathrm{Tl}, 53,54 \mathrm{PbTe}: \mathrm{Ti}, 6 \mathrm{Mg} 2 \mathrm{Sn}: \mathrm{Ag} .55$ This method further allows to distinguish between localized (e.g. PbTe:Ti) and delocalized (e.g. PbTe:Tl) resonant level,7 as well as between resonant and non-resonant impurity behavior.55 More details about the Bloch spectral functions can be found in Refs. 49 and 50. 


\section{Results and discussion}

\subsection{Electronic band structure}

The electronic structure of the binary SnTe compound was widely discussed in literature.56-58 SnTe is a narrow-band-gap semiconductor, with a band gap $E_{g}=0.18 \mathrm{eV}$ at $300 \mathrm{~K} .16$ Its valence bands are mainly characterized by a light and a heavy-hole band, just like in its sister compound PbTe. Strictly speaking, the light and heavy-hole bands are not two different bands, but two maxima of the last valence band, one occurring at the L point and the second in the so-called $\Sigma$ point (located half way between $\Gamma$ and $\mathrm{K}$ in the $k_{z}=0$ plane). The experimentally-determined energy offset between these two maxima is about $0.3-0.4 \mathrm{eV} .16$

As the presence of Sn vacancies is an inherent property of SnTe, making it naturally heavily hole doped, Sn vacancies were added in our electronic structure calculations to simulate the system close to the experimental one. Figure 1a shows the density of states of SnTe containing $0.32 \%$ of Sn vacancies. Sn vacancies act, as expected, as two-hole acceptors, shifting the Fermi level into the valence bands. This vacancy concentration corresponds to a hole concentration of $10_{20} \mathrm{~cm}-3$, as typically observed in real SnTe samples, and is very close to the experimental value estimated in $\mathrm{Sn} 1.03 \mathrm{Te}(0.25 \%$ corresponding to a hole concentration of $8.0 \times 1019 \mathrm{~cm}-3) .59$ As a first approximation, vacancies do not visibly modify the electronic structure of SnTe, the effect of which is thus mostly a rigid-band-like shift in $E_{F}$. The situation is radically different when considering the DOS of SnTe doped with $0.5 \%$ of In and without Sn vacancies (Figure 1b) where the In-induced formation of the resonant level can be clearly seen. The carrier concentration of this system is $7.9 \times 1019 \mathrm{~cm}-3$, as In is a monovalent acceptor (that is, in a In $1+$ valence state). The mechanism of In (usually a trivalent element with the $5 s_{2} 5 p_{1}$ valence shell configuration) becoming an acceptor against $\mathrm{Sn}$ (divalent in $\mathrm{SnTe}$ as shown by 
Mössbauer spectroscopy in Ref 59, 60 and 61) is similar to that of In in Bi (Ref. 62) or Tl in PbTe.53,54,63,64 One $5 s$ electron of In forms a hyper-deep (HD) state in the low-energy region, below the $p$-like main valence band (VB), which bounds one $5 p$ electron from Te taken from the main VB. One $5 p$ electron of In contributes to the main valence band block, and the second $5 s$ electron of In forms the resonant state. As a result, upon substituting In for $\mathrm{Sn}$, the main VB is left with one electron less, making In an acceptor. The main difference with the Bi:In system is that In does not create there a resonant level near $E_{F}$.

The formation of the DOS "hump", in agreement with previous reports,4,7,64 is related to the presence of In atoms. As shown in Figure 1c where the partial DOS of In atoms is shown, the DOS peak formed by In $5 s$ states defines the resonant behavior of In in SnTe. When the In concentration increases to $2 \%$ (Figure 1d), the DOS "hump" broadens and the hole concentration increases.

We further considered the DOS of $0.5 \%$ In-doped SnTe containing $0.32 \%$ of Sn vacancies (Figures 1e and 1f,) in order to analyze the influence of Sn vacancies on the formation of the resonant level. Three effects are observed: (i) RL on In is also formed in the presence of vacancies; (ii) $E_{F}$ is deeper in the valence bands due to additional holes given by the vacancies; (iii) RL is slightly smeared resulting in a slightly lower partial In DOS (Figure 1f). As in such a case, in calculations, three "atoms" ( $\mathrm{Sn}$, In and vacancy) occupy the same crystallographic site, one might expect some additional RL smearing effect due to enhanced disorder, compared to the vacancy-free compound.

To analyze the influence of vacancy and RL on the electronic bands, Bloch spectral density functions (BSF) were computed for the same compositions. Figure 2 shows the twodimensional projections of BSF plotted in a way similar to the usual dispersion relations in several BZ directions, with the color marking the value of BSF. Figure 2a presents BSF of $p$ type SnTe containing $0.32 \%$ of Sn vacancy. The general features of the electronic structure of 
SnTe are in agreement with previous density-functional calculations and experimental studies.56-58 What is worth noting is that the minimum of the conduction band appears to be slightly off the L point, which is better seen when bands in the off-high-symmetry $\mathrm{L}-\Sigma$ direction are plotted. While signatures of this effect may be seen in other studies, (e.g. in Ref. 56 in the $\mathrm{W}-\mathrm{L}$ direction), no direct comparison is possible, since bands in the $\mathrm{L}-\Sigma$ direction were not previously computed in literature. Moreover, this effect may be related to the inaccuracy of the local density approximation as well. In contrast to PbTe, we are not aware of any calculation going beyond the local density approximation (LDA) or the generalized gradient approximation (GGA) for SnTe, that is, using the GW method. Nevertheless, this potential error would be more important for $n$-type compounds than the $p$-type compositions studied herein. The L- and $\Sigma$-band separation is here about $0.15 \mathrm{eV}$ which is lower than for the energy offset obtained in prior studies. This underestimation is a direct consequence of the inaccuracy of the local density approximation. Of note, this error will manifest in calculations of the effective mass $m_{D O S}^{*}$ as a function of the hole concentration, discussed below, as a too early increase. We note that a similar situation was reported for PbTe.54

As far as the effect of vacancies on the band structure is concerned, we may observe a minor band smearing, leaving sharp, well-defined bands (Figure 2a). In contrast, as shown in Figure $2 \mathrm{~b}$, strong band smearing is observed for $0.5 \%$ In-doped SnTe containing $0.32 \%$ of Sn vacancies. This effect is obviously related to the presence of resonant In impurities and is very similar to what has been observed in PbTe:Tl.53,54 In the presence of In-induced RL, electronic states are shifted towards the valence band edge and form a "cloud" around the $\mathrm{L}-\Sigma$ band. However, these electronic states do not form an isolated impurity band due to hybridization between resonant In and host SnTe states. These conclusions hold true when the In concentration reaches $2 \%$ (Figure 2c): the number of electronic states around the $\mathrm{L}-\Sigma$ band increases (which is responsible for the larger DOS "hump" in Figure 1d), band smearing is 
enhanced without forming an impurity band. To quantify the level of hybridization between the resonant and host states, the In atomic contribution to the $\operatorname{DOS}\left(E_{F}\right)$ has been calculated. The results indicate that, even for $2 \%$ In, it does not exceed $10 \%$. Thanks to this hybridization, the localization of the RL or the formation of a narrow impurity band is avoided. The strong band smearing, induced by RL, causes enhanced carrier scattering, which will dominate the transport properties of the system at low temperatures. By analyzing the width of the spectral function $\Delta$ (that is, the width of the smeared band), the electronic life time $\tau=\hbar / \Delta$ may be estimated. For $k$-points in the middle of $\mathrm{L}-\Sigma$ path, this approach yields $\tau \sim 3 \times 10-14 \mathrm{~s}$ for $0.5 \%$ In-doped SnTe and $\tau \sim 3 \times 10-13 \mathrm{~s}$ for SnTe containing $0.32 \%$ of Sn vacancies. Similar magnitude of the carrier life time was obtained for PbTe:Tl.54 These results highlight the importance of carrier scattering on the resonant level. Since these calculations correspond to $T=0 \mathrm{~K}$, this predicted reduction of the lifetime due to RL, compared to self-doped SnTe, will lead to enhanced residual resistivities at low temperatures, which could be experimentally verified, if very good quality samples of pristine SnTe are available in order to avoid microstructure-related effects. Note that the link between the evolution of the residual resistivity and the carrier lifetimes should remain qualitative due to the fact that a direct relation between carrier lifetime and relaxation time that appears in transport quantities is not straightforward. Such increase in the residual resistivity was observed for the PbTe:Tl system when compared to PbTe:Na,54 and may be used as an independent, experimental verification of the existence of the RL. Extending the present measurements to liquid-helium temperatures will be the subject of a forthcoming study.

Enhanced carrier scattering due to the presence of RL is also responsible for the larger, concentration-dependent resistivity of SnTe:In samples at $T>300 \mathrm{~K}$, as discussed below in the experimental section. It should be stressed here that scattering on RL, which is strong in SnTe:In samples, is much less temperature-dependent than scattering of carriers on phonons. Because of this difference, the transport properties of "resonant" samples are expected to exhibit weaker 
dependence on temperature than rigid-band-impurities-doped samples. At higher temperatures where the carrier relaxation time due to scattering on phonons is on the same order of magnitude as the scattering on $\mathrm{RL}(\tau \sim 10-14 \mathrm{~s})$, differences in $\rho$ for samples with and without resonant impurities is not as large as the difference in carrier life times at $T=0 \mathrm{~K}$ might have suggested. The ratio of the electrical resistivities for the most ( $2 \%$ In) and least ( $0 \%$ In) resistive samples in our studies (see below) changes from 5.4 at $300 \mathrm{~K}$ to 1.3 at $800 \mathrm{~K}$. This weaker temperature sensitivity is expected to give rise to enhanced peak power factor and $Z T$ values, but also to higher $Z T$ over a broader temperature range, yielding higher average $Z T$, which is an important parameter for practical applications.

A qualitative link between the RL-induced increase in DOS and its effect on thermopower can be inferred from the calculation of the DOS effective mass $m_{D O S}^{*}$ of the studied materials, using the method discussed in Refs. 54 and 65. The results of these computations as a function of the carrier concentration (obtained by rigidly shifting $E_{F}$ in the DOS computed for the actual material's composition) are shown in Figure 3. Absolute mass values are affected by the LDA inaccuracy in two ways, as can be analyzed for the "self-doped" SnTe case. Firstly, the lightband mass, which has been determined to be about $0.168 m_{0}\left(m_{0}\right.$ is the bare electron mass $)$ in prior studies, is about $0.4 m_{0}$ in our case. Secondly, the rapid increase in $m_{D O S}^{*}$ near $7 \times 1019 \mathrm{~cm}$ 3 in the case of SnTe with $0.32 \%$ Sn vacancies is caused by the too high position of the top of the heavy-hole $\Sigma$ band, the effect of which should appear at higher hole concentrations. Nevertheless, the region of interest is the region before the heavy-hole band is reached. In spite of these inaccuracies, a relative increase of the $m_{D O S}^{*}$ due to In doping is clearly observed. Hence, higher In concentrations will result in higher $m_{D O S}^{*}$, and ultimately, in higher thermopower values.

Summarizing this theoretical part, KKR-CPA calculations confirm the formation of a resonant level when In is substituted on the Sn site in SnTe. Most importantly, this effect is not 
affected by the presence of Sn vacancies, which have nevertheless a small, but visible effect on the details of the RL. Electronic bands are smeared but no separated impurity band is formed which would have been detrimental to electrical conduction. Finally, the In-induced RL increases the DOS effective mass, proportionally to the In concentration. As we will see below, the experimental results lend strong support to these predictions.

\subsection{Crystal structure and sample purity}

The phase purity of the densified Sn1.03-xIn $x$ Te samples has been assessed by PXRD (Figure 4). These patterns show that all the synthesized samples are single phased within the detection limits of PXRD. All the peaks can be indexed to the NaCl-type cubic structure of SnTe described in the $F m \overline{3} m$ space group. However, two additional peaks at $30.7^{\circ}$ and $32.1^{\circ}$ that correspond to elemental Sn can be observed. The presence of these peaks is consistent with the Sn excess systematically introduced in the initial compositions and located at the grain boundaries, as shown by transmission electron microscopy.61 The absence of In-rich secondary phases up to the highest In content of $2 \%$ is in agreement with prior studies that reported a much higher solubility limit of In of around $40 \% .37,38,44$ The lattice parameter of the $x=0.02$ sample

( $a=6.3197(2) \AA)$, inferred from Rietveld refinements against the PXRD data, is slightly lower than that determined for the $x=0.0$ sample $(a=6.3242(2) \AA)$. For intermediate In concentrations below $1 \%$, no evident shift in the lattice parameter was observed due to the very low In contents investigated. These results are consistent with prior studies evidencing that In substitution exerts a chemical pressure on the unit cell with $a$ weakly shrinking for In concentrations up to about $9 \%$ before decreasing more rapidly up to the $40 \%$ substitution level.37,38 


\subsection{Electronic transport properties}

Figures $5 \mathrm{a}$ and $5 \mathrm{~b}$ show the temperature dependences of the electrical resistivity and thermopower, respectively. Both the $\rho(T)$ and $\alpha(T)$ data of the $x=0$ sample are consistent with prior studies revealing a metallic behavior up to about $600 \mathrm{~K}$ above which a stronger rise with temperature is observed.11-33 This peculiar effect has been attributed to the contribution of the heavy-hole valence band which coexists with a light-hole valence band that dominates transport at lower temperatures.66-68 This general trend is hardly affected upon substituting In for Sn up to about $x=0.0045$. Above this value, further increasing the In concentration tends to lessen the superlinear variation above $600 \mathrm{~K}$. While observable on the $\rho(T)$, this effect is more clearly evidenced in the $\alpha(T)$ data where the high-temperature increase is no longer visible in the $x=$ 0.02 sample.

The most remarkable effect induced by In substitution mainly occurs near room temperature where both $\alpha$ and $\rho$ significantly increase with increasing the In content in a stepwise manner. Given the very low In concentrations introduced, this finding provides strong experimental evidence that these concomitant enhancements arise from an In-induced RL. The fact that $\alpha$ and $\rho$ continuously evolve upon increasing the In content can be explained by the local DOS enhancement predicted by our electronic band structure calculations. While the DOS peak governs the increase in $\alpha$, both the decrease in the carrier lifetime and the increase in the DOS effective mass induced by the RL lead to a strong reduction of the hole mobility (see below) and hence, of the $\rho$ values.

Despite this detrimental impact on the $\rho$ values, the best compromise near room temperature is achieved in the $x=0.075$ and 0.01 samples which show the highest power factor (Figure 6). Upon warming, $P$ no longer evolves linearly with the In content above $550 \mathrm{~K}$ due to the significant increase in $\alpha$ experienced notably by the $x=0$ and 0.005 samples. 
Nevertheless, except for the $x=0.02$ sample for which $P$ is clearly lowered, all the samples exhibit slightly enhanced, similar $P$ values which are shifted towards higher temperatures with a maximum value of $1.8 \times 10_{-3} \mathrm{~W}$ m-1 K-2 achieved at $800 \mathrm{~K}$. These results are consistent with those obtained by Zhang et al.33 for the series $\operatorname{Sn}_{1-x} \operatorname{In} x$ Te. The enhancement of the power factor at high temperatures, as we already mentioned, is possible due to the fact that phonon scattering and resonant level scattering are on the same order of magnitude near room temperature. Upon warming, the scattering on RL is less temperature-dependent, although still strong, which allows to take advantage from the increase in $\alpha$.

The influence of In on the Hall hole concentration $p_{H}$ and Hall mobility $\mu_{H}$ has been probed by Hall effect measurements performed at $300 \mathrm{~K}$. Of note, $p_{H}$ is related to the chemical hole concentration $p$ by the relation $p_{H}=r_{H} p$ where $r_{H}$ is the Hall factor usually taken equal to unity. However, it was shown in $\mathrm{Pb}_{1-x} \mathrm{Tl}_{x} \mathrm{Te}$ that the $\mathrm{RL}$ affects this factor in degenerate samples, the extent to which depends on the concentration of the resonant impurity and temperature.69 The deviations from unity were predicted to be the largest at low temperatures and low $\mathrm{Tl}$ concentrations while the effect on $r_{H}$ remains moderate near room temperature. In the present $\operatorname{Sn}_{1.03-x} \operatorname{In}_{x}$ Te series, assuming $r_{H} \approx 1$, and hence $p_{H} \approx p$, near $300 \mathrm{~K}$ should therefore provide a reasonable estimate of $p$. The dependence of $p_{H}$ and $\mu_{H}$ on $x$, shown in Figure 7, confirms a rapid and strong reduction in $\mu_{H}$ with increasing $x$ despite the very low doping levels considered herein. Meanwhile, $p_{H}$ tends to slightly but continuously increase with $x$ suggesting that In behaves as an acceptor impurity in SnTe in agreement with our electronic band structure calculations. This is further confirmed by the slope of $p_{H}$ versus the concentration of In atoms per $\mathrm{cm}-3$ (not shown) which corresponds to about 0.6 holes per In atom. The evolution of these two parameters with $x$ confirms that the reduction of $\mu_{H}$ is the main factor that drives the observed increase in $\rho$. Such a reduction in $\mu_{H}$ is expected in the presence of a RL, as mentioned above. 
One straightforward and convenient way of revealing the resonant nature of an impurity atom is tied to the carrier concentration dependence of the thermopower known as the IoffePisarenko plot. The resonant behavior manifests itself by significant deviations from the conventional dependence followed by a rigid-band-like impurity. In the present case, this analysis is complicated by the presence of two valence bands that govern the transport of SnTe and the number of band parameters that need to be taken into account. Pioneering studies on SnTe by Brebrick et al. (Refs. 66, 67 and 68) have shown that a two-valence-band model using a combination of a parabolic heavy-hole band and non-parabolic light-hole band provides a good description of the experimental data over two orders of magnitude of the hole concentration. As shown in Figure 8, the comparison of our experimental data at $300 \mathrm{~K}$ with the theoretical curve show that all the data of the In-substituted samples strongly deviate from the predicted variation. For the $x=0.0$ sample, as discussed elsewhere, 61 the deviation from theory may come from the single set of band parameters used to calculate the Pisarenko curve, some of which may likely vary with varying the Sn vacancy concentration. For the Incontaining samples, a steep rise in the $\alpha$ values with increasing the In content is observed around $1020 \mathrm{~cm}-3$ while the model predicts only little variations around this hole concentration. Moreover, the peak value of $\alpha$ seems to be enhanced and shifted towards lower hole concentration compared to the extremum predicted slightly below $10_{21} \mathrm{~cm}-3$. The marked difference between a rigid-band model and experiment provides another strong evidence of the resonant character of In in $\mathrm{Sn} 1.03 \mathrm{Te}$.

As a consistency check, we tried to determine whether changes in the band parameters used to calculate the theoretical curve could explain the observed trend. The results, presented in Figure S1 in the ESI file, show that the density-of-states effective masses of the heavy-hole and light-hole bands are the two main parameters that control the peak in $\alpha$ and its position. However, an increase in the magnitude of the peak and its shift towards lower hole 
concentration are observed when both effective masses significantly decrease, in contradiction with the evolution of $m_{D O S}^{*}$ predicted by our electronic band structure calculations. It seems therefore difficult to reconcile these theoretical results with the experimental data without considering the presence of an In-induced resonant RL. Since the main role of the RL may be qualitatively understood as an increase in the density-of-states effective mass, its influence on the hole concentration dependence of the thermopower cannot be captured by the abovementioned two-valence-band model using a single set of band parameters.

\subsection{Thermal transport properties}

Figure 9 shows the temperature dependence of the total thermal conductivity $\kappa$. For all samples, $\kappa$ decreases with increasing temperature and tends to saturate above $700 \mathrm{~K}$ to values ranging between 2.1 and $2.7 \mathrm{~W} \mathrm{m-1} \mathrm{K-1.} \mathrm{In} \mathrm{contrast} \mathrm{to} \mathrm{the} \mathrm{roughly} \mathrm{similar} \mathrm{values} \mathrm{observed} \mathrm{at} \mathrm{the} \mathrm{highest}$ temperatures, $\kappa$ strongly varies with the In concentration near room temperature from $7.9 \mathrm{~W} \mathrm{~m}$ $1 \mathrm{~K}-1$ in the $x=0$ sample down to $3.9 \mathrm{~W} \mathrm{~m}-1 \mathrm{~K}-1$ in the $x=0.02$ sample. While the temperature dependences of $\kappa$ up to $x=0.0045$ are very similar, clear differences emerge for higher In concentrations. In addition to an overall decrease with temperature strongly lessened, a crossover near $500 \mathrm{~K}$ is clearly visible. Above this temperature, $\kappa$ no longer decreases with increasing $x$ for $x \geq 0.0045$ but surprisingly shows the opposite trend. This reversed variation with $x$ likely reflects significant differences in the electronic thermal conductivity $\kappa_{e}$. This hypothesis is justified by the low In concentrations and the small difference in molar mass between $\mathrm{Sn}$ and In which are expected to result in weak mass, binding force and strain field differences induced by In alloying. Thus, according to the relation proposed by Callaway and Klemens to determine the evolution of $\kappa_{p h}$ in solid solutions, 70,71 the difference between $\kappa_{p h}$ of pure $\mathrm{Sn}_{1.03} \mathrm{Te}$ and that of the In-containing samples should be very small. 
Although disentangling the electronic and lattice contributions to $\kappa$ by estimating $\kappa_{e}$ via the Wiedemann-Franz law $\kappa_{e}=L T / \rho$ would shed further light on this behavior, this subtraction requires to obtain a correct estimate of the Lorenz number $L$. However, this parameter is difficult to determine in SnTe due to its strong dependence on the details of the electronic band structure and on temperature. For most of SnTe-based compounds, a simple single-parabolic-band model with acoustic phonon scattering is usually used to determine the temperature dependence of $L$. While this model provides reasonable estimates of $\kappa_{p h}$ in samples exhibiting low hole concentrations, it fails to yield consistent results for heavily hole-doped samples. For the present series, neither a single-parabolic-band model nor a two-valence-band model with temperature-dependent band parameters (Figure S2 in the ESI file) provides a satisfying approach to determine $L$. Both models tend to overestimate $L$ giving rise to large differences in $\kappa_{p h}$ among the samples (Figure S3 in the ESI file), which are not expected given the very low Sn vacancy concentration in Sn1.03Te (estimated to be around 0.25\%, Ref. 61) and the narrow In concentration range investigated herein. In addition, none of these models capture well the change in the transport regime observed above $500 \mathrm{~K}$, the temperature above which the second heavy-valence band plays a significant role on the electronic conduction and hence, on $L$.

Considering as valid the hypothesis that the effect of alloying with In up to $x=0.02$ hardly affects $\kappa_{p h}$, the strong increase in $\rho$ with $x$ then provides an alternative approach to estimate the temperature dependence of $L$ in the present case. The weak temperature dependence of $\kappa$ and the absence of significant upturn in $\alpha$ above $500 \mathrm{~K}$ in the $x=0.02$ sample further suggests a negligible role of the second heavy-hole valence band. Assuming that the $L$ values calculated for the $x=0.02$ sample yield a correct estimate of $\kappa_{p h}$, we used the same $\kappa_{p h}$ values to calculate the temperature dependence of $\kappa_{e}$ expected for the other samples. 
Following this approach, the expected temperature dependences of $L$ were derived from $\kappa_{e}(T)$ for all samples (Figure 10). Except for the $x=0.02$ sample, the $L$ values are strongly temperature dependent and drop well below the non-degenerate limit of $1.5 \times 10-8 \mathrm{~V}_{2} \mathrm{~K}-2$ expected for acoustic phonon scattering. For the $x=0.0035$ sample, $L$ even reaches $0.8 \times 10-8 \mathrm{~V}_{2}$ $\mathrm{K}-2$ above $600 \mathrm{~K}$. The upturn in $L$ observed at high temperatures for low doping levels is likely an artifact, possibly due to minority carrier activation across the band gap of $0.18 \mathrm{eV}$. These results show that the Lorenz numbers should be significantly lower than those predicted by simple transport models to obtain consistent estimates of $\kappa_{p h}$ across the entire series of samples. Such low values likely reflect the multiband character of the valence bands which has been shown to give rise to significant deviations from the $L$ values expected in single-band systems.72,73 In addition, because the relaxation time strongly depends on energy when the chemical potential nears the resonant state due to its significant width in energy (see Figure 2), scattering of the carriers may become strongly inelastic, thereby leading to significant variations in the Lorenz number. In particular, this effect has been discussed in the PbTe:Tl compounds for which, large deviations of $L$ from its degenerate limit has been shown to occur depending on the position of the chemical potential with respect to the resonant-band center.69 The fact that the largest deviations of $L$ are observed in the $x=0.0035$ sample, which features the most pronounced RL effect, is an intriguing indication that the RL has a strong impact on the Lorenz number. It is thus likely that these two combined effects are responsible for the anomalous Lorenz numbers inferred and provide a consistent explanation of the large differences in the $L$ values observed from sample to sample despite the very narrow In composition range covered in this series. 


\subsection{Dimensionless thermoelectric figure of merit $Z T$}

The temperature dependence of the ZT values, shown in Figure 11, evidences a clear increase at $800 \mathrm{~K}$ upon substituting In for Sn. A maximum $Z T$ value of 0.75 is achieved for $x=0.0035$ which represents an increase of $35 \%$ compared to the $Z T$ value of the pristine sample Sn1.03Te. This peak value is lower than that achieved by Zhang et al.33 due to the lower total thermal conductivity achieved by a reduction of the grain size via ball-milling. In our case, the significant increase observed in the $\rho$ values with increasing $x$ is the main limiting factor that prevents higher peak $Z T$ values from being achieved at higher In concentrations. However, interestingly, the average $Z T$ value $Z T_{a v g}$, an important parameter for practical applications determined by integrating with respect to temperature over an extended temperature range, 74 is maximized in the $x=0.0035$ sample. Compared to the pristine sample where $Z T_{\text {avg }}=0.45$ over the $600-800 \mathrm{~K}$ temperature range, a concentration as low as $x=0.0035$ is sufficient to increase this value to 0.58 over the same temperature range (Table 1). This result shows that the weak temperature dependence of the RL helps to enhance $Z T_{a v g}$ even though the increase in the peak $Z T$ value is moderate.

\section{Conclusions}

We reported on a detailed joint theoretical and experimental study of the influence of In on the high-temperature thermoelectric properties of $\mathrm{Sn} 1.03 \mathrm{Te}$. KKR-CPA calculations have demonstrated the formation of a resonant state upon substituting In for Sn. The RL formation is only weakly affected by the presence of Sn vacancies, which have a small, but visible effect on the details of the resonant state. The computed Bloch spectral functions show that electronic bands are strongly smeared in SnTe:In but without forming a separated impurity band. The In- 
induced resonant state increases the DOS effective mass, proportionally to the In concentration. Our extensive collection of experimental data obtained on samples with In concentrations spanning the range $0 \leq x \leq 0.02$ are all consistent with the presence of a RL disturbing the valence band structure of $\mathrm{Sn} 1.03 \mathrm{Te}$. Despite the very low In contents of the present series, significant differences were observed with a stepwise increase with $x$ in the thermopower and electrical resistivity. The latter trend originates from the strong impact of the RL on the hole mobility, the $x$ dependence of which cannot be captured within a rigid-band-like scenario. Compared to prior studies on In-substituted SnTe, the maximum power factor is shifted towards higher In concentrations due to the Sn self-compensation used herein. This difference highlights the needs to adjust the carrier concentration in RL-containing systems so that the position of the chemical potential is optimized against that of the RL. Although the beneficial influence of the RL observed at $300 \mathrm{~K}$ is somewhat lessened at $800 \mathrm{~K}$, an increase in the $Z T$ values is nevertheless obtained with a peak value of 0.75 at $800 \mathrm{~K}$ for $x=0.0035$, which represents an increase of $35 \%$ compared to $\mathrm{Sn} 1.03 \mathrm{Te}$. Another important consequence of the RL for practical applications is its weak temperature dependence that yields a higher average $Z T$ value compared to $\mathrm{Sn} 1.03 \mathrm{Te}$. The progressive increase in the In content has also a profound influence on the thermal transport with a clear evolution in the temperature dependence of the total thermal conductivity as $x$ increases. In particular, the strong increase in $\rho$ with $x$ enabled us to shed light on the difficult task to correctly evaluate the Lorenz number in SnTe due to the multiband nature of its valence bands. Based on a set of reasonable assumptions, our analysis unraveled very low Lorenz numbers that even drop below the non-degenerate limit. This result highlights that, despite the high degeneracy of the charge carrier gas, the multiband character of the electronic bands and the presence of the RL combine to significantly lower the Lorenz numbers above $300 \mathrm{~K}$, making it difficult to adequately estimate the lattice thermal conductivity. Finally, the sensitivity of the transport properties to the concentration of the RL impurity further evidences 
that the combination of an RL impurity with a co-doping element should be carefully chosen to avoid the shift of the chemical potential away from the RL or the disappearance of the RL due to band structure modifications induced by the second element.

Electronic Supplementary Information (ESI) available.

\section{Conflicts of interest}

The authors declare no competing financial interest.

\section{Acknowledgements}

B. W. was supported by the National Science Center (Poland), project No. 2017/26/E/ST3/00119. J. T. was supported by the AGH UST statutory tasks no. 11.11.220.01/5 within subsidy of the Ministry of Science and Higher Education. 


\section{References}

1 Goldsmid, H. J. in Thermoelectric Refrigeration; Springer: New York, 1964.

2 Thermoelectrics and its Energy Harvesting, edited by D. M. Rowe (CRC Press, 2012).

3 J. P. Heremans, V. Jovovic, E. S. Toberer, A. Saramat, K. Kurosaki, A. Charoenphakdee, S. Yamanaka and G. J. Snyder, Science, 2008, 321, 554-557.

4 D. G. Mahan and J. O. Sofo, Proc. Natl. Acad. Sci. U. S. A., 1996, 93, 7436-7439.

5 J. P. Heremans, B. Wiendlocha and A. M. Chamoire, Energy Environ. Sci., 2012, 5, 55105530.

6 B. Wiendlocha, Appl. Phys. Lett., 2014, 105, 133901.

7 B. Wiendlocha, K. Kutorasinski, S. Kaprzyk, J. Tobola, Scripta Materialia, 2016, 111, 33-38.

8 C. M. Jaworski, V. Kulbachinskii and J. P. Heremans, Phys. Rev. B, 2009, 80, 233201.

9 B. Wiendlocha, J. Electron. Mater., 2016, 45, 3515-3531.

${ }_{10}$ B. Wiendlocha, J.-B. Vaney, C. Candolfi, A. Dauscher, B. Lenoir and J. Tobola, Phys. Chem. Chem. Phys., 2018, 20, 12948-12957.

11 G. Tan, L-D. Zhao, F. Shi, J. W. Doak, S.-H. Lo, H. Sun, P. Wang, C. Wolverton, V. P. Dravid, C. Uher, and M. G. Kanatzidis, J. Am. Chem. Soc., 2014, 136, 7006-7017.

12 G. Tan, F. Shi, S. Hao, H. Chi, L-D. Zhao, C. Uher, C. Wolverton, V. P. Dravid, and M. G. Kanatzidis, J. Am. Chem. Soc., 2015, 137, 5100-5112.

13 G. Tan, F. Shi, S. Hao, H. Chi, T. P. Bailey, L-D. Zhao, C. Uher, C. Wolverton, V. P. Dravid, and M. G. Kanatzidis, J. Am. Chem. Soc., 2015, 137, 11507-11516.

${ }_{14}$ G. Tan, F. Shi, J. W. Doak, H. Sun, L-D. Zhao, P. Wang, C. Uher, C. Wolverton, V. P. Dravid and M. G. Kanatzidis, Energy Environ. Sci., 2015, 8, 267-277.

15 G. Tan, W. G. Zeier, F. Shi, P. Wang, G. J. Snyder, V. P. Dravid, and M. G. Kanatzidis, Chem. Mater., 2015, 27, 7801-7811. 
16 M. Zhou, Z. M. Gibbs, H. Wang, Y. Han, C. Xin, L. Li and G. J. Snyder, Phys. Chem. Chem. Phys., 2014, 16, 20741-20748.

17 J. He, X. Tan, J. Xu, G.-Q. Liu, H. Shao, Y. Fu, X. Wang, Z. Liu, J. Xu, H. Jiang and J. Jiang, J. Mater. Chem. A, 2015, 3, 19974-19979.

18 A. Banik, U. S. Shenoy, S. Anand, U. W. Waghmare and K. Biswas, Chem. Mater., 2015, 27, $581-587$.

19 Z. Zhou, J. Yang, Q. Jiang, Y. Luo, D. Zhang, Y. Ren, X. He and J. Xin, J. Mater. Chem. A, 2016, 4, 13171-13175.

20 L-D. Zhao, X. Zhang, H. Wu, G. Tan, Y. Pei, Y. Xiao, C. Chang, D. Wu, H. Chi, L. Zheng, S. Gong, C. Uher, J. He and M. G. Kanatzidis, J. Am. Chem. Soc., 2016, 138, 2366-2373.

${ }_{21}$ R. Al Rahal Al Orabi, J. Hwang, C.-C. Lin, R. Gautier, B. Fontaine, W. Kim, J.-S. Rhyee, D. Wee and M. Fornari, Chem. Mater., 2017, 29, 612-620.

22 L. Zhang, J. Wang, Z. Cheng, Q. Sun, Z. Li and S. Dou, J. Mater. Chem. A, 2016, 4, 79367942.

23 A. Banik, B. Vishal, S. Perumal, R. Datta and K. Biswas, Energy Environ. Sci., 2016, 9, 20112019.

24 R. Al Rahal Al Orabi, N. A. Mecholsky, J. Hwang, W. Kim, J.-S. Rhyee, D. Wee and M. Fornari, Chem. Mater., 2016, 28, 376-384.

25 L. Wang, X. Tan, G. Liu, J. Xu, H. Shao, B. Yu, H. Jiang, S. Yue and J. Jiang, ACS Energy Lett., 2017, 2, 1203-1207.

26 W. Li, L. Zheng, B. Ge, S. Lin, X. Zhang, Z. Chen, Y. Chang and Y. Pei, Adv. Mater., 2017, 29, 1605887.

27 N. Wang, D. West, J. Liu, J. Li, Q. Yan, B.-L. Gu, S. B. Zhang, W. Duan, Phys. Rev. B, 2014, 89, 045142.

28 L. Zheng, W. Li, S. Lin, J. Li, Z. Chen and Y. Pei, ACS Energy Lett., 2017, 2, 563-568. 
29 L. Hu, Y. Zhang, H. Wu, J. Li, Y. Li, M. McKenna, J. He, F. Liu, S. J. Pennycook, X. Zeng, Adv. Energy Mater., 2018, 8, 1802116.

30 J. Q. Li, S. Huang, Z. P. Chen, Y. Li, S. H. Song, F. S. Liu, W. Q. Ao, Phys. Chem. Chem. Phys., 2017, 19, 28749-28755.

31 S. Roychowdhury, U. S. Shenoy, U. V. Waghmare and K. Biswas, J. Mater. Chem. C, 2017, $\mathbf{5}, 5737-5748$

32 D. Ibrahim, V. Ohorodniichuk, C. Candolfi, C. Semprimoschnig, A. Dauscher and B. Lenoir, ACS Omega, 2017, 2, 7106-7111.

33 Q. Zhang, B. Liao, Y. Lan, K. Lukas, W. Liu, K. Esfarjani, C. Opeil, D. Broido, G. Chen and Z. Ren, Proc. Natl. Acad. Sci. U. S. A., 2013, 110, 13261-13266.

34 A. S. Erickson, J.-H. Chu, M. F. Toney, T. H. Geballe and I. R. Fisher, Phys. Rev. B, 2009, 79, 024520.

35 V. K. Maura, P. Srivastava and S. Patnaik, Euro. Phys. Lett., 2014, 108, 37010.

36 M. Novak, S. Sasaki, M. Kriener, K. Segawa and Y. Ando, Phys. Rev. B, 2013, 88, 140502.

37 N. Haldolaarachchige, Q. Gibson, W. Xie, M. Bormann Nielsen, S. Kushwaha and R. J. Cava, Phys. Rev. B, 2016, 93, 024520.

38 R. D. Zhong, J. A. Schneeloch, X. Y. Shi, Z. J. Xu, C. Zhang, J. M. Tranquada, Q. Li and G. D. Gu, Phys. Rev. B, 2013, 88, 020505.

${ }_{39}$ G. Balakrishnan, L. Bawden, S. Cavendish and M. R. Lees, Phys. Rev. B, 2013, 87, 140507. 40 M. P. Smylie, H. Claus, W.-K. Kwok, E. R. Louden, M. R. Eskildsen, A. S. Sefat, R. D. Zhong, J. Schneeloch, G. D. Gu, E. Bokari, P. M. Niraula, A. Kayani, C. D. Dewhurst, A. Snezhko, and U. Welp, Phys. Rev. B, 2018, 97, 024511.

${ }_{41}$ K. Kobayashi, Y. Ai, H. O. Jeschke and J. Akimitsu, Phys. Rev. B, 2018, 97, 104511.

42 T. Sato, Y. Tanaka, K. Nakayama, S. Souma, T. Takahashi, S. Sasaki, Z. Ren, A. A. Taskin, K. Segawa and Y. Ando, Phys. Rev. Lett., 2013, 110, 206804. 
43 S. Sasaki, Z. Ren, A. A. Taskin, K. Segawa, L. Fu and Y. Ando, Phys. Rev. Lett., 2012, 109, 217004.

${ }_{44}$ C. Zhang, X.-G. He, H. Chi, R. Zhong, W. Ku, G. Gu, J. M. Tranquada and Q. Li, Phys. Rev. $B, 1998,98,054503$.

${ }_{45}$ Z. Xu, J. A. Schneeloch, R. D. Zhong, J. A. Rodriguez-Rivera, L. W. Harriger, R. J. Birgeneau, G. D. Gu, J. M. Tranquada and G. Xu, Phys. Rev. B, 2015, 91, 054522.

46 P. Giraldo-Gallo, P. Walmsley, B. Sangiorgio, S. C. Riggs, R. D. McDonald, L. Buchauer, B. Fauqué, Chang Liu, N. A. Spaldin, A. Kaminski, K. Behnia, and I. R. Fisher, Phys. Rev. Lett., 2018, 121, 207001.

${ }_{47}$ S. A. Nemov and Y. I. Ravich, Sov. Phys. Usp., 1998, 41, 735-759.

48 V. I. Kaidanov and Y. I. Ravich, Sov. Phys. Usp., 1985, 28, 31-53.

${ }_{49}$ H. Ebert, D. Ködderitzsch, and J. Minár, Rep. Prog. Phys., 2011, 74, 096501.

50 J. S. Faulkner and G. M. Stocks, Phys. Rev. B, 1980, 21, 3222-3244.

51 H. Ebert, The Munich SPR-KKR package, version 7.7.3 http://ebert.cup.unimuenchen.de/SPRKKR, 2018.

52 S. H. Vosko, L. Wilk, and M. Nusair, Can. J. Phys., 1980, 58, 1200-1211.

53 B. Wiendlocha, Phys. Rev. B, 2013, 88, 205205.

54 B. Wiendlocha, Phys. Rev. B, 2018, 97, 205203.

55 S. Kim, B. Wiendlocha, H. Jin, J. Tobola and J. P. Heremans, J. Appl. Phys., 2014, 116, 153706.

${ }_{56}$ P. B. Littlewood, B. Mihaila, R. K. Schulze, D. J. Safarik, J. E. Gubernatis, A. Bostwick, E. Rotenberg, C. P. Opeil, T. Durakiewicz, J. L. Smith, and J. C. Lashley, Phys. Rev. Lett., 2010, 105, 086404.

57 S. Rabii, Phys. Rev., 1969, 182, 821-828.

${ }_{58}$ C. M. I. Okoye, J. Phys.: Condens. Matter, 2002, 14, 8625-8637. 
59 W. Keune, Phys. Rev. B, 1974, 10, 5057-5062.

60 V. Fano, G. Fedeli and I. Ortalli, Solid State Commun., 1977, 22, 467-470.

61 D. Ibrahim, C. Candolfi, S. Migot, J. Ghanbaja, A. Dauscher, B. Malaman, C. Semprimoschnig and B. Lenoir, Phys. Rev. Mater., 2019.

62 H. Jin, B. Wiendlocha and J. P. Heremans, Energy Environ. Sci., 2015, 8, 2027-2040.

63 S. Ahmad, K. Hoang, and S. D. Mahanti, Phys. Rev. Lett., 2006, 96, 056403.

64 X. J. Tan, G. Q. Liu, J. T. Xu, H. Z. Shao, J. Jiang and H. C. Jiang, Phys. Chem. Chem. Phys., 2016, 18, 20635-20639.

65 K. Kutorasinski, B. Wiendlocha, J. Tobola, and S. Kaprzyk, Phys. Rev. B, 2014, 89, 115205.

66 R. F. Brebrick and A. J. Strauss, Phys. Rev., 1963, 131, 104-110.

${ }_{67}$ R. F. Brebrick and E. Gubner, J. Chem. Phys., 1962, 36, 1283-1289.

68 R. F. Brebrick, J. Phys. Chem. Solids, 1963, 24, 27-36.

69 L. V. Prokof'eva, A. A. Shabaldin, V. A. Korchagin, S. A. Nemov and Yu. I. Ravich, Semiconductors, 2008, 42, 1161-1170.

70 J. Callaway, Phys. Rev., 1959, 113, 1046-1051.

71 P. G. Klemens, Phys. Rev., 1960, 119, 507-509.

72 R. W. McKinney, P. Gorai, V. Stevanovic and E. S. Toberer, J. Mater. Chem. A, 2017, 5, $17302-17311$.

73 M. Thesberg, H. Kosina and N. Neophytou, Phys. Rev. B, 2017, 95, 125206.

74 H. S. Kim, W. Liu, G. Chen, C.-W. Chu and Z. Ren, Proc. Natl. Acad. Sci. U. S. A., 2015, 112, 8205-8210. 


\section{Tables}

Table 1. Average $Z T$ values calculated over the $300-600 \mathrm{~K}$ and $600-800 \mathrm{~K}$ temperature ranges for all samples.

\begin{tabular}{c|c|c}
\hline \multirow{2}{*}{ In concentration } & \multicolumn{2}{|c}{ Average $Z T$} \\
\cline { 2 - 3 } & $300-600 \mathrm{~K}$ & $600-800 \mathrm{~K}$ \\
\hline 0.0 & 0.09 & 0.46 \\
0.0005 & 0.11 & 0.51 \\
0.0015 & 0.12 & 0.51 \\
0.0025 & 0.13 & 0.49 \\
0.0035 & 0.15 & 0.58 \\
0.0045 & 0.15 & 0.52 \\
0.0075 & 0.15 & 0.50 \\
0.0100 & 0.15 & 0.49 \\
0.0200 & 0.13 & 0.41 \\
\hline \hline
\end{tabular}




\section{Figure Captions}

Figure 1. Densities of states as a function of the energy for SnTe: a) with $0.32 \%$ Sn vacancies; b) doped with $0.5 \%$ In, with partial In DOS in panel c); d) doped with $2 \%$ In; e) doped with 0.5\% In and with $0.32 \%$ Sn vacancy, with partial In DOS in panel f). In panels a), b), d) and e), atomic contributions are weighted by their concentration. In panels c) and f), the DOS is given per In atom.

Figure 2. Two-dimensional projection of the Bloch spectral functions in high symmetry directions for SnTe: a) containing $0.32 \%$ Sn vacancies; b) doped with $0.5 \%$ In and with $0.32 \%$ Sn vacancy; c) doped with $2 \%$ In. Colors mark BSF values, plotted in logarithmic scale. Black color corresponds to BSF values higher than 300 a.u. In resonant impurity leads to the smearing of the $\mathrm{L}-\Sigma$ valence band near the band edge.

Figure 3. Hole dependence of the density of states effective mass $m_{D O S}^{*}$ for SnTe containing vacancies and doped with In. Resonant doping visibly increases the effective mass of the light band. The rapid increase in $m_{D O S}^{*}$, observed in the case of $\mathrm{SnTe}+0.32 \% \mathrm{Sn}$ vacancies near $7 \times 1019 \mathrm{~cm}-3$, is caused by the too high position of the top of the " $\Sigma$ band", which should appear at significantly higher hole concentrations.

Figure 4. PXRD patterns of the samples in the series $\operatorname{Sn} 1.03-x \operatorname{In} x \operatorname{Te}(0 \leq x \leq 0.02)$. For clarity, all the patterns of the In-containing samples have been vertically offset. The asterisks mark the position of the reflections of elemental $\mathrm{Sn}$. 
Figure 5. Temperature dependence of a) the electrical resistivity $\rho$ and b) thermopower $\alpha$ of the $\operatorname{Sn} 1.03-x \operatorname{In} x$ Te samples $(0 \leq x \leq 0.02)$. The solid lines are guides to the eye.

Figure 6. Power factor $\alpha^{2} / \rho$ as a function of temperature for the series $\operatorname{Sn}_{1.03-x} \operatorname{In}_{x} \operatorname{Te}(0 \leq x \leq$ 0.02). The solid lines are guides to the eye.

Figure 7. Variations in the hole concentration $p$ and Hall mobility $\mu_{H}$ as a function of the In concentration $x$. The solid lines are guides to the eye.

Figure 8. Ioffe-Pisarenko plot showing the thermopower $\alpha$ as a function of the hole concentration $p$ at $300 \mathrm{~K}$. The solid black curve corresponds to the theoretical dependence determined using a two-valence-band model. For comparison purposes, the values reported by Zhang et al. (Ref. 33) have been added.

Figure 9. Total thermal conductivity $\kappa$ as a function of the temperature for the series $\mathrm{Sn}_{1.03-}$ ${ }_{x} \operatorname{In} x \operatorname{Te}(0 \leq x \leq 0.02)$. The solid lines are guides to the eye.

Figure 10. Temperature dependence of the Lorenz number $L$ for the $\operatorname{Sn}_{1.03-x} \operatorname{In}{ }_{x} T e$ samples $(0 \leq$ $x \leq 0.02)$ predicted using the Wiedemann-Franz law. The electronic thermal conductivity $\kappa_{e}$ was evaluated by subtracting from $\kappa$ the lattice thermal conductivity $\kappa_{p h}$ of the $x=0.02$ sample assumed to be constant across the series. The solid lines are guides to the eye.

Figure 11. Dimensionless thermoelectric figure of merit $Z T$ as a function of temperature for the $\operatorname{Sn} 1.03-x \operatorname{In} x$ Te samples $(0 \leq x \leq 0.02)$. The solid lines are guides to the eye. 

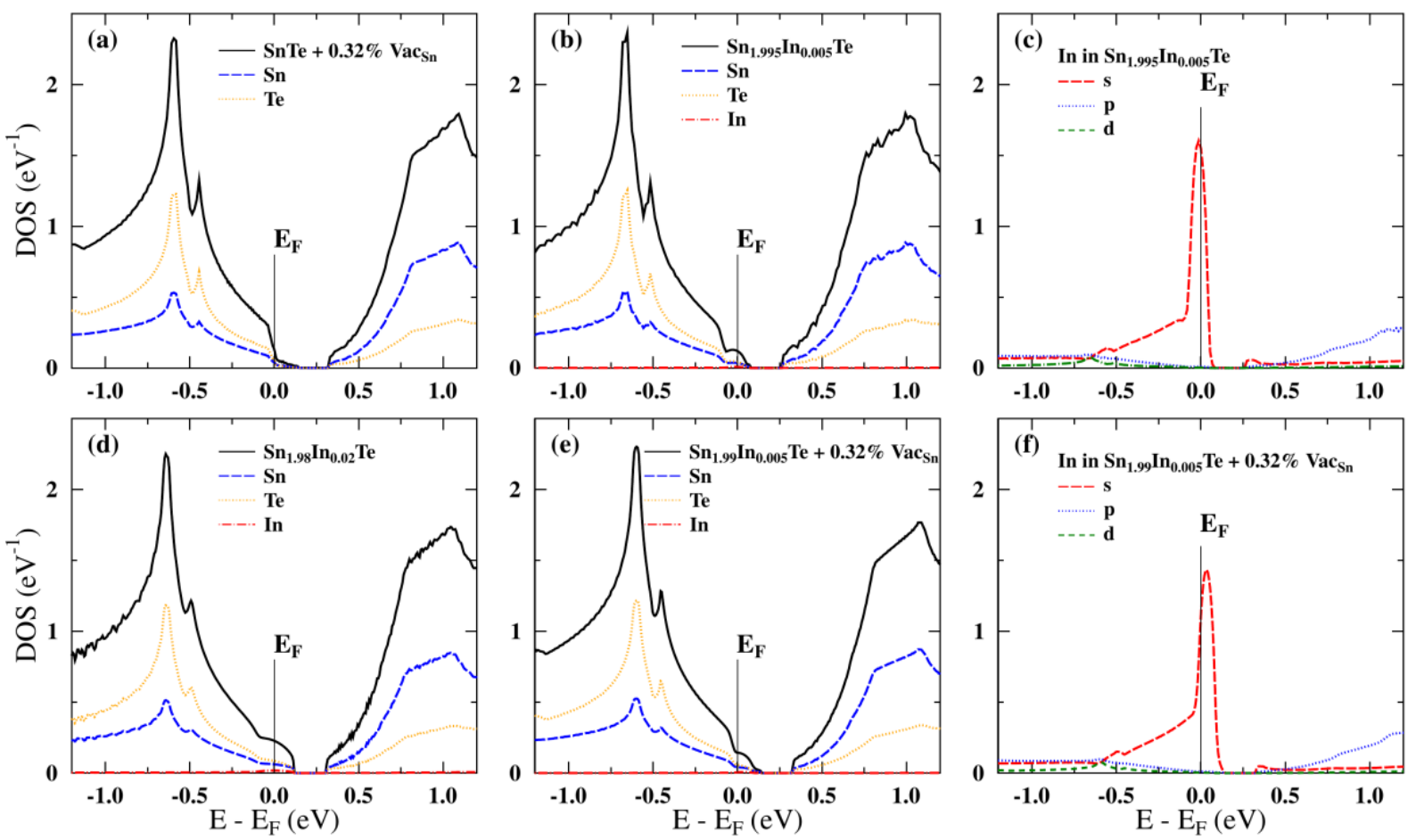

Figure 1 

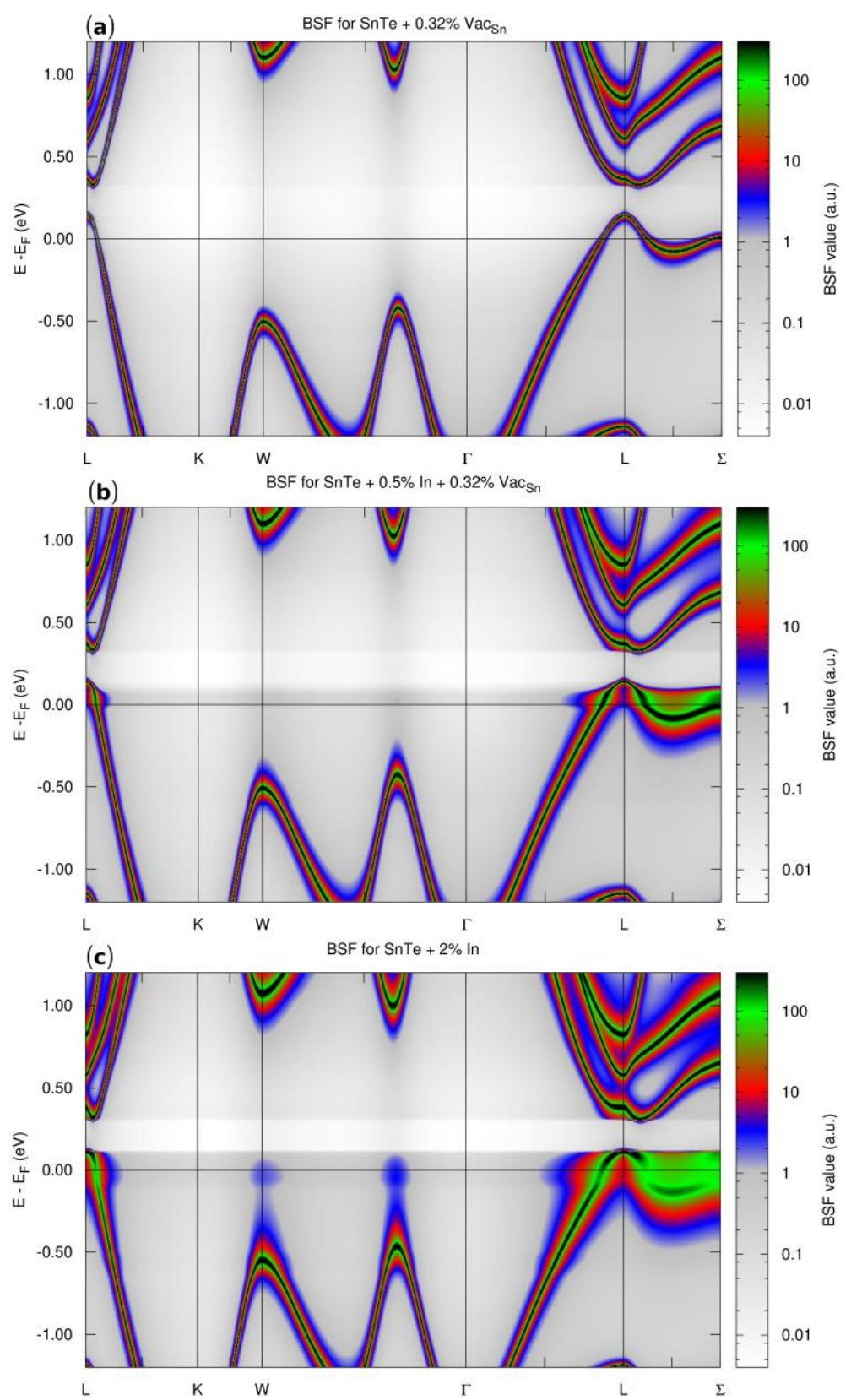

Figure 2 


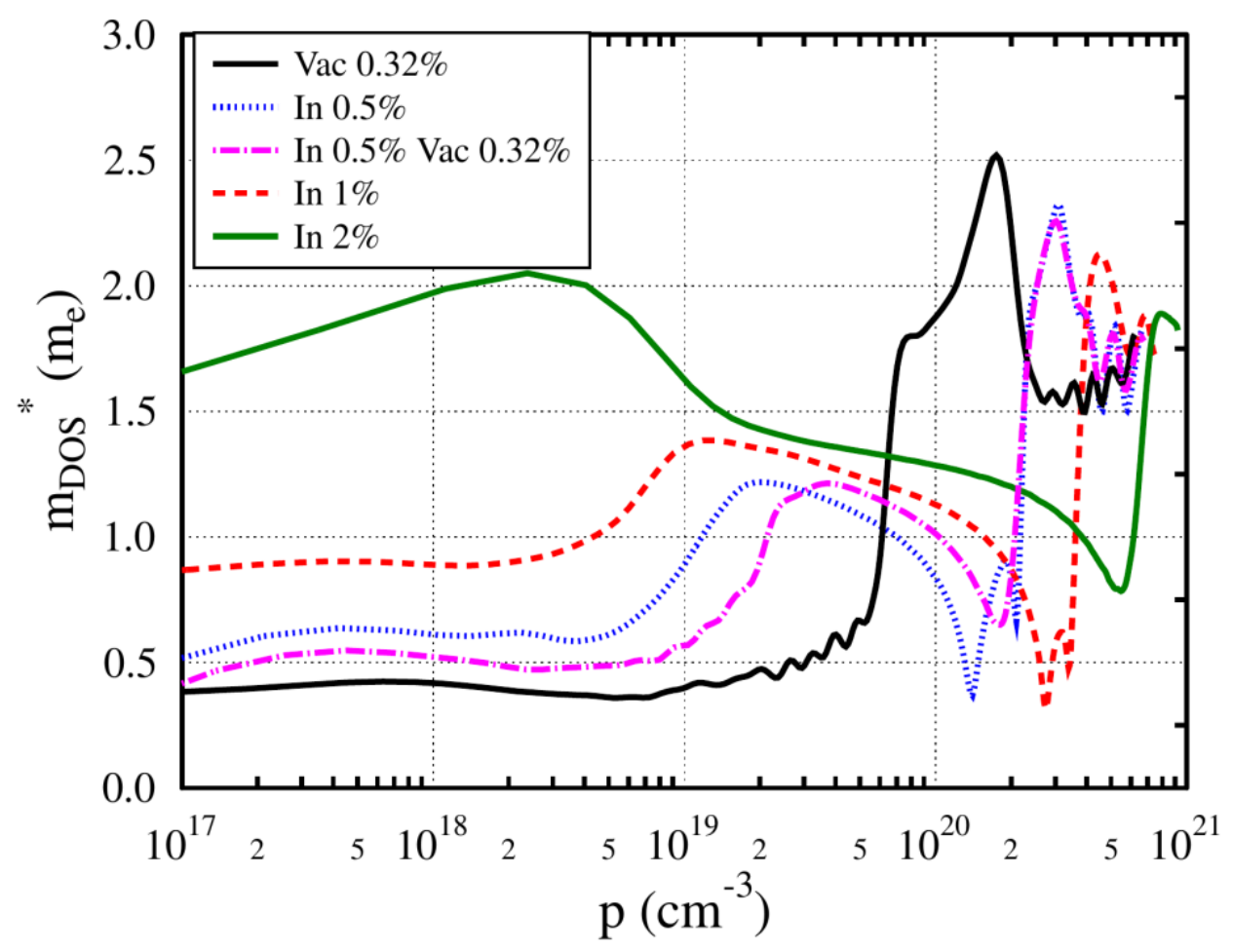

Figure 3 


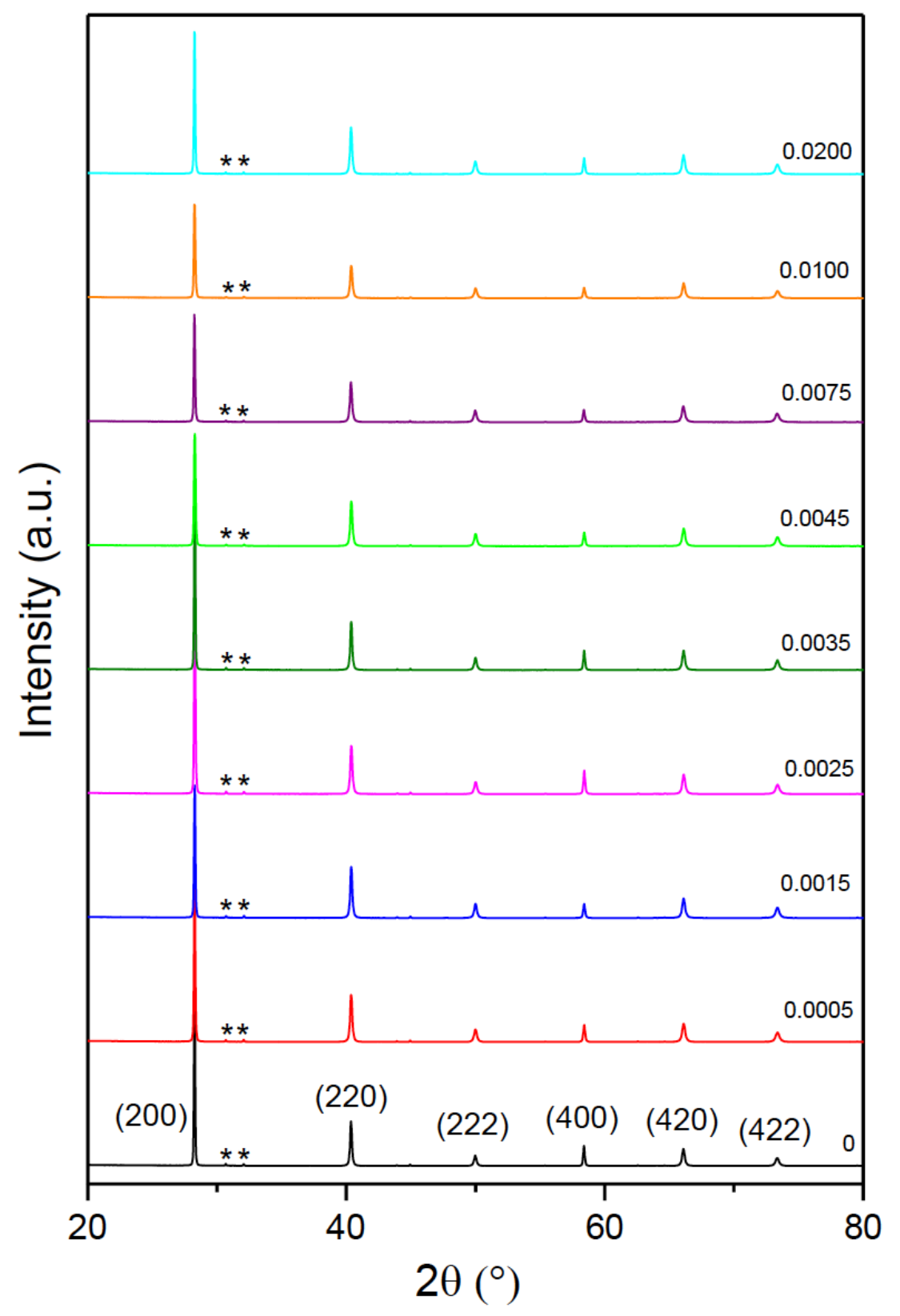

Figure 4 
a)

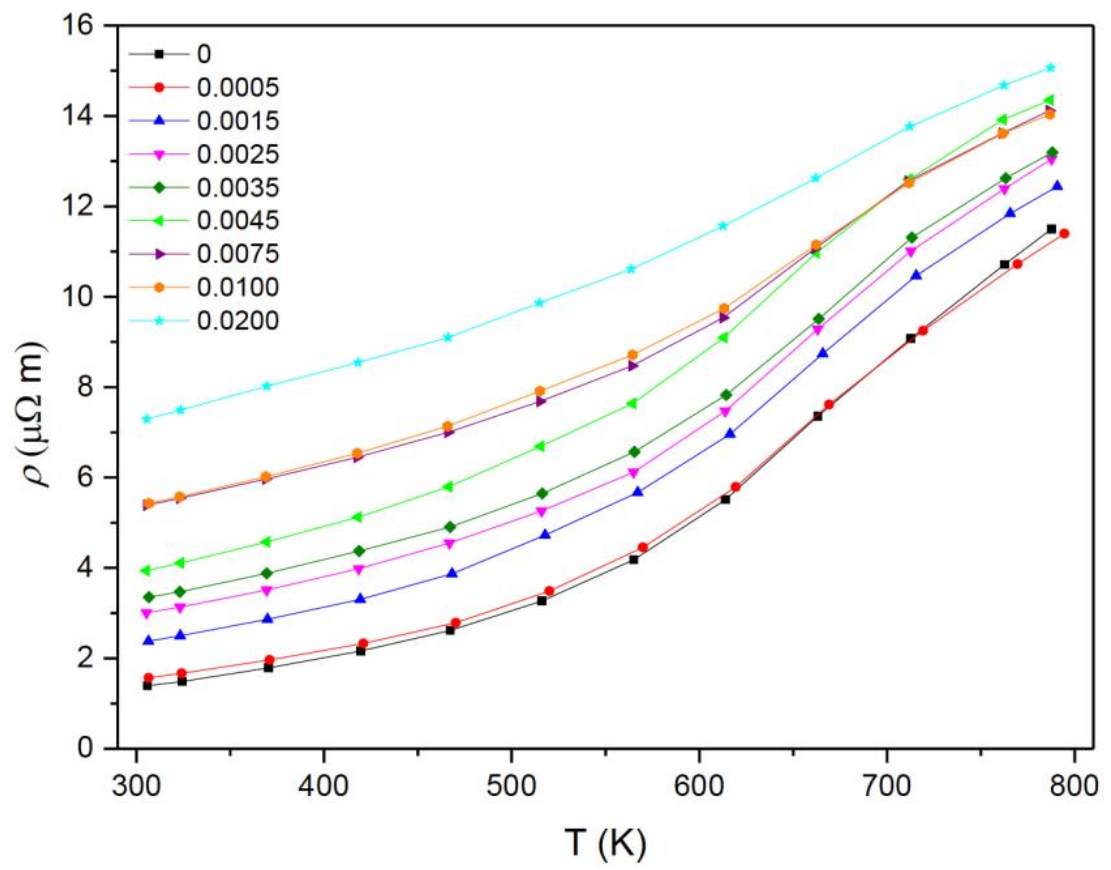

b)

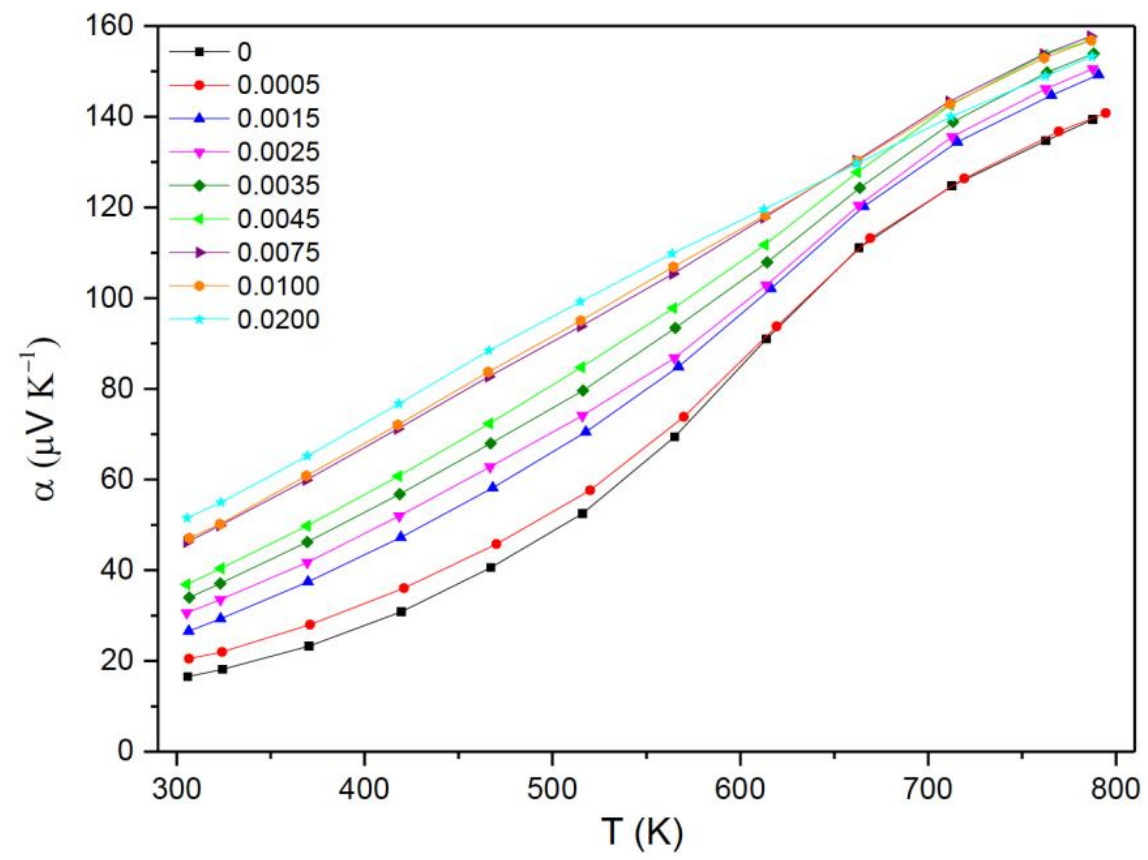

Figure 5 


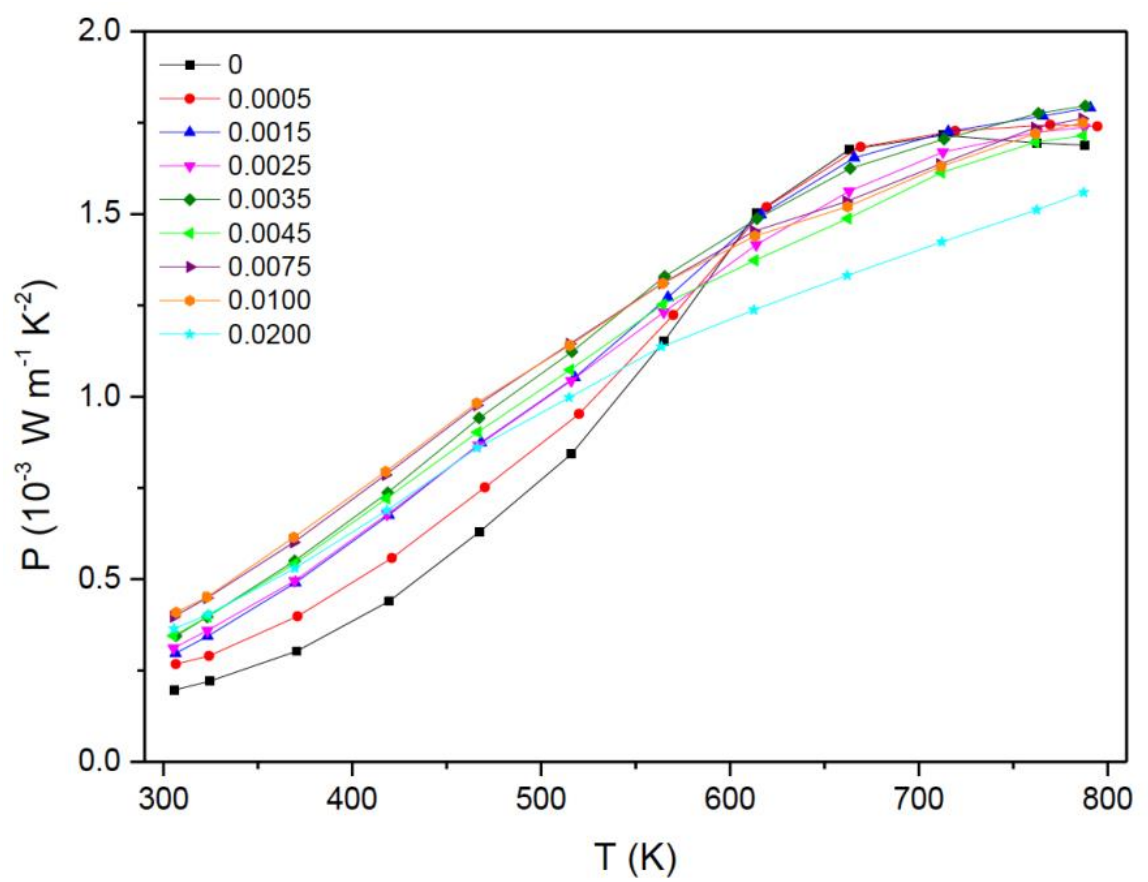

Figure 6 


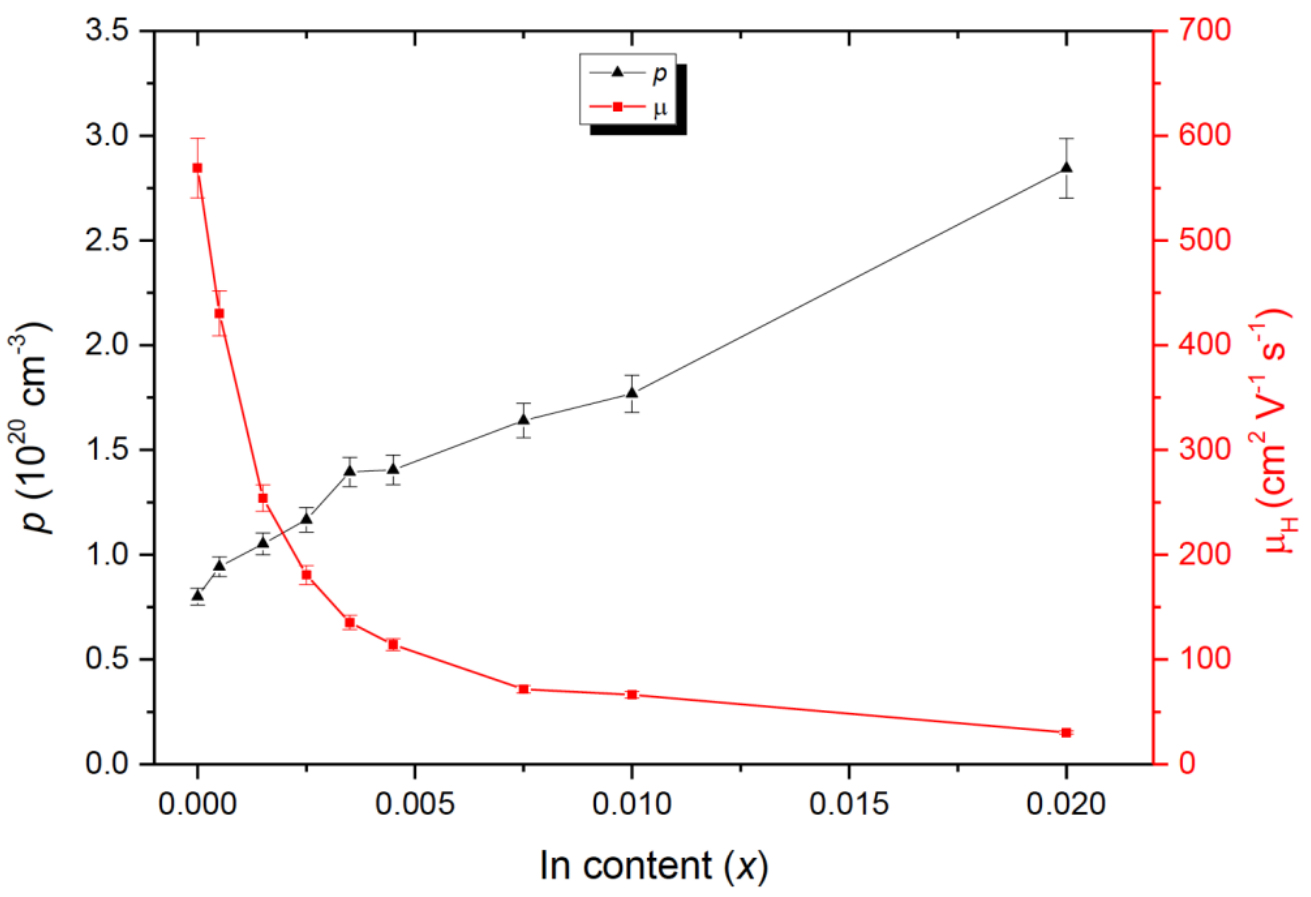

Figure 7 


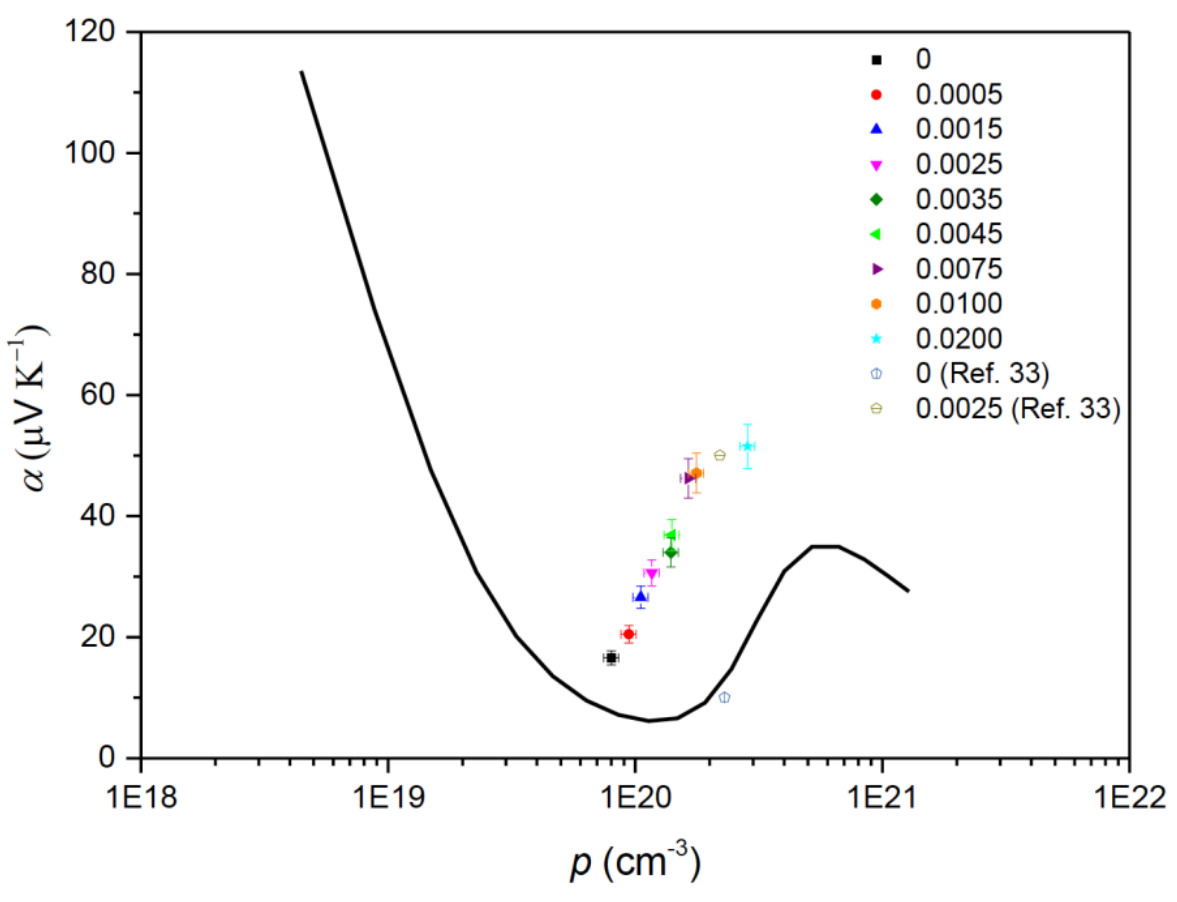

Figure 8 


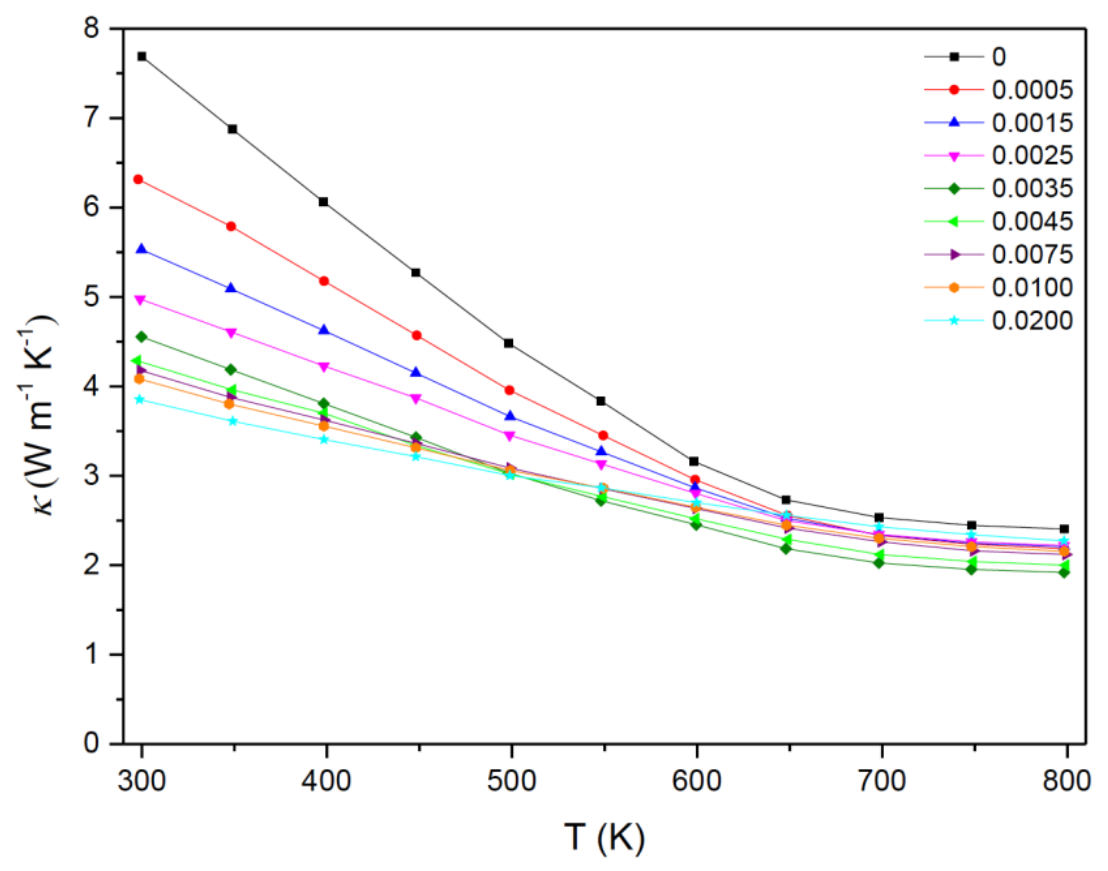

Figure 9 


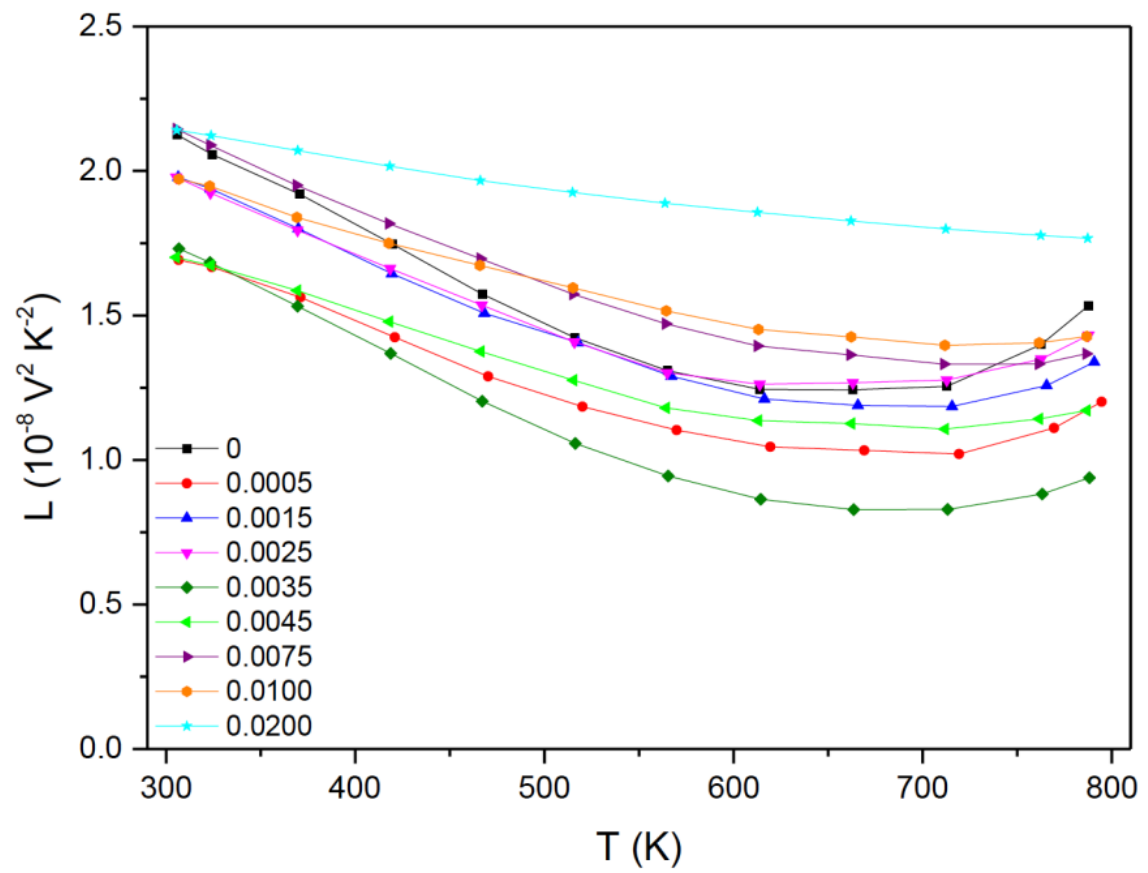

Figure 10 


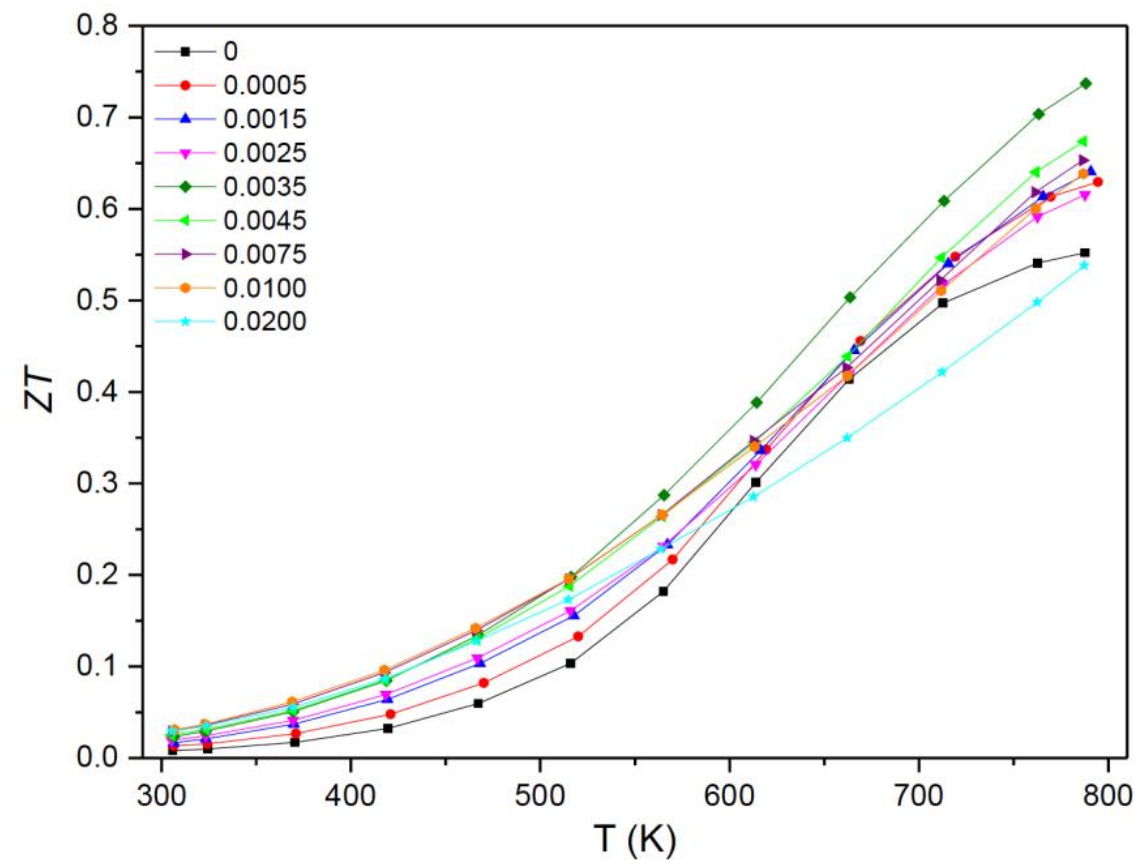

Figure 11 